\title{
ON ARTIN FORMALISM FOR THE CONJECTURE OF BLOCH AND KATO
}

\author{
DAVID BURNS
}

\begin{abstract}
We prove that the Tamagawa Number Conjecture of Bloch and Kato satisfies a natural 'Artin formalism' and then describe several explicit applications of this result.
\end{abstract}

\section{Introduction}

Fix a number field $k$, an algebraic closure $k^{c}$ of $k$ and a motive $M$ that is defined over $k$. For each finite extension $E$ of $k$ in $k^{c}$ write $M_{E}$ for the corresponding motive $h^{0}(\operatorname{Spec}(E))(0) \otimes_{h^{0}(\operatorname{Spec}(k))(0)} M$ defined over $E$.

As a concrete example of this construction, we recall that if $M$ is the $r$-fold Tate twist $h^{n}(X)(r)$ of the motive arising from the cohomology in degree $n$ of a variety $X$ defined over $k$, then $M_{E}$ identifies with $h^{n}\left(X_{/ E}\right)(r)$, where $X_{/ E}$ denotes $X$ regarded as defined over $E$.

In this paper, we shall first prove (in Theorem 3.1) that as $E$ varies over all finite extensions of $k$ in $k^{c}$ the Tamagawa Number Conjecture for $M_{E}$ that was formulated by Bloch and Kato in [4], and then later reformulated and extended by Fontaine and Perrin-Riou in [13], satisfies the same 'Artin formalism' that has played a key role in recent work of Dokchitser and Dokchitser [9, 10] and Bartel [1] regarding abelian varieties and unit groups. The method that we use to prove this result is purely Galois-cohomological in nature and can be used in exactly the same way to prove the analogous (but finer) result for motives with coefficients in any given number field but, except for Remark 3.2, we prefer for clarity of exposition to omit any explicit mention of such cases.

We then discuss (in Section 4) several concrete applications of Theorem 3.1 and also describe a more conceptual approach to the theory of 'regulator constants' that was introduced in [10].

\section{The Bloch-Kato Conjecture}

2.1. Virtual objects. Throughout this paper we shall use Deligne's formalism of virtual objects and so we now quickly introduce some of the necessary notation.

We fix an associative unital noetherian ring $R$. Modules over $R$ are to be understood, unless explicitly stated otherwise, as left modules.

We write $V(R)$ for the Picard category of virtual objects over $R$ that is defined by Deligne in [8]. For any finitely generated projective $R$-module $P$ we write $[M]_{R}$ for the associated object of $V(R)$. We write $(X, Y) \mapsto X \cdot Y$ for the product in $V(R)$ and define $\mathbf{1}_{R}$ to be the unit object $[0]_{R}$ of $V(R)$. For each object $X$ of $V(R)$ we fix an

Received by the editors February 17, 2012. 
'inverse object' $X^{-1}$ and a 'contraction morphism' $\mathrm{ev}_{X}: X \cdot X^{-1} \rightarrow \mathbf{1}_{R}$ in $V(R)$. We write $\mathcal{P}_{0}$ for the Picard category with unique object $\mathbf{1}_{\mathcal{P}_{0}}$ and the group $\operatorname{Aut}_{\mathcal{P}_{0}}\left(\mathbf{1}_{\mathcal{P}_{0}}\right)$ trivial.

We let $D(R)$ denote the derived category of $R$-modules and $D^{\mathrm{p}}(R)$ the full triangulated subcategory of $D(R)$ comprising complexes that are quasi-isomorphic to a bounded complex of finitely generated projective $R$-modules. We often (and without explicit comment) identify an $R$-module $M$ with the object $M[0]$ of $D(R)$ that is equal to $M$ in degree 0 and is zero in all other degrees.

If $R$ is a discrete valuation ring of characteristic 0 and $F$ is a field that contains $R$, then for every object $C$ of $D^{\mathrm{p}}(R)$ and every morphism $t: F \otimes_{R}[C]_{R} \rightarrow \mathbf{1}_{F}$ in $V(F)$ we write $\chi^{\text {ref }}(C, t)$ for the element of the relative algebraic $K$-group $K_{0}(R, F)$ that corresponds to the isomorphism class of the pair $\left([C]_{R}, t\right)$ under the explicit isomorphism of abelian groups $\pi_{0}\left(V(R) \times_{V(F)} \mathcal{P}_{0}\right) \cong K_{0}(R, F)$ that is described in [6, Prop. 2.8]. (This element $\chi^{\mathrm{ref}}(C, t)$ is often referred to as the 'refined Euler characteristic' of the pair $(C, t)$.) For the reader's convenience, we have recalled some of the basic properties of these constructions in an Appendix.

2.2. Review of the Bloch-Kato Conjecture. We continue to use the general notation introduced in Section 2.1. For any Galois extension of fields $F^{\prime} / F$ we also set $G_{F^{\prime} / F}:=\operatorname{Gal}\left(F^{\prime} / F\right)$.

We fix a finite Galois extension $K$ of $k$ in $k^{c}$ and a finite set of places $S$ of $k$ containing the set $S_{\infty, k}$ of all archimedean places, all which ramify in $K / k$ and all at which $M$ has bad reduction. For each intermediate field $E$ of $K / k$ we write $S_{E}$ for the set of places of $E$ above those in $S$ and for each prime $p$ we write $S_{p}$ for the union of $S$ and all places of $k$ that divide $p$. We fix a full $G_{k^{c} / k^{-}}$-stable $\mathbb{Z}_{p}$-lattice $T_{p}$ in the $p$-adic realization of $M$ and for each field $E$ as above we set $T_{p, E}:=T_{p} \otimes_{\mathbb{Z}_{p}} \prod_{E \rightarrow k^{c}} \mathbb{Z}_{p}$, endowed with the diagonal left action of $G_{k^{c} / k}$ and also, if $E / k$ is Galois, with the obvious commuting left action of the group ring $\mathbb{Z}_{p}\left[G_{E / k}\right]$ (on the second factor in the tensor product).

For any finite set of places $\Sigma$ of $E$ that contains $S_{\infty, E}$ we write $\mathcal{O}_{E, \Sigma}$ for the subring of $E$ comprising elements that are integral at all places outside $\Sigma$ and we abbreviate $\mathcal{O}_{E, S_{\infty, k}}$ to $\mathcal{O}_{E}$. We now assume that $E / k$ is Galois and recall that the compactly supported étale cohomology complex $R \Gamma_{c \text {,ét }}\left(\mathcal{O}_{k, S_{p}}, T_{p, E}\right)$ of $T_{p, E}$ on $\operatorname{Spec}\left(\mathcal{O}_{k, S_{p}}\right)$ is an object of $D^{\mathrm{p}}\left(\mathbb{Z}_{p}\left[G_{E / k}\right]\right)$ (by, for example, [12, Th. 5.1]). We further recall that, under certain standard conjectures (see Remark 2.1 below), the approach of [6, Section 3.4] constructs a canonical object $\Xi(M, E / k)$ of $V\left(\mathbb{Q}\left[G_{E / k}\right]\right)$ together with a canonical morphism in $V\left(\mathbb{Q}_{p}\left[G_{E / k}\right]\right)$

$$
\vartheta_{p}(M, S, E / k): \mathbb{Q}_{p} \otimes_{\mathbb{Q}} \Xi(M, E / k) \rightarrow \mathbb{Q}_{p} \otimes_{\mathbb{Z}_{p}}\left[R \Gamma_{c, \text { ét }}\left(\mathcal{O}_{k, S_{p}}, T_{p, E}\right)\right]_{\mathbb{Z}_{p}\left[G_{E / k}\right]}
$$

and a canonical morphism in $V\left(\mathbb{R}\left[G_{E / k}\right]\right)$

$$
\vartheta_{\infty}(M, E / k): \mathbb{R} \otimes_{\mathbb{Q}} \Xi(M, E / k) \rightarrow \mathbf{1}_{\mathbb{R}\left[G_{E / k}\right]} .
$$

For each prime $p$, we fix an isomorphism $j: \mathbb{C} \cong \mathbb{C}_{p}$ and then write

$$
t_{j}^{\mathrm{BK}}(M, S, E / k): \mathbb{C}_{p} \otimes_{\mathbb{Z}_{p}}\left[R \Gamma_{c, \text { ét }}\left(\mathcal{O}_{k, S_{p}}, T_{p, E}\right)\right]_{\mathbb{Z}_{p}\left[G_{E / k}\right]} \rightarrow \mathbf{1}_{\mathbb{C}_{p}\left[G_{E / k}\right]}
$$

for the composite morphism $\left(\mathbb{C}_{p} \otimes_{\mathbb{R}, j} \vartheta_{\infty}(M, E / k)\right) \circ\left(\mathbb{C}_{p} \otimes_{\mathbb{Q}_{p}} \vartheta_{p}(M, S, E / k)^{-1}\right)$. 
If $E=K=k$, so that $S$ can be any finite set of places of $E$ containing both $S_{\infty, E}$ and all places at which $M$ has bad reduction, then we abbreviate $\Xi(M, E / k)$, $\vartheta_{p}(M, S, E / k), \vartheta_{\infty}(M, E / k)$ and $t_{j}^{\mathrm{BK}}(M, S, E / k)$ to $\Xi(M), \vartheta_{p}(M, S), \vartheta_{\infty}(M)$ and $t_{j}^{\mathrm{BK}}(M, S)$, respectively. In particular, in this way we obtain for each intermediate field $E$ of $K / k$ an element of $K_{0}\left(\mathbb{Z}_{p}, \mathbb{C}_{p}\right)$ by setting

$$
\chi_{j}^{\mathrm{BK}}\left(M_{E}\right):=\chi^{\mathrm{ref}}\left(R \Gamma_{c, \text { ét }}\left(\mathcal{O}_{E, \Sigma_{p}}, T_{p}\right), t_{j}^{\mathrm{BK}}\left(M_{E}, \Sigma\right)\right),
$$

where $\Sigma$ is any finite set of places of $E$ containing both $S_{\infty, E}$ and all places at which $M_{E}$ has bad reduction. This element $\chi_{j}^{\mathrm{BK}}\left(M_{E}\right)$ is independent of the choices of the set $\Sigma$ and lattice $T_{p}$ (by, for example, [6, Lem. 5]) and can, and will, be regarded as a free rank one $\mathbb{Z}_{p}$-sublattice of $\mathbb{C}_{p}$ via the natural identification $K_{0}\left(\mathbb{Z}_{p}, \mathbb{C}_{p}\right) \cong \mathbb{C}_{p}^{\times} / \mathbb{Z}_{p}^{\times}$. Further, if the 'Coherence hypothesis' of [6, Section 3.3] is valid for the motive $M_{E}$, then there exists an object $\Xi\left(M_{E}\right)_{\mathbb{Z}}$ of $V(\mathbb{Z})$ with $\mathbb{Q} \otimes_{\mathbb{Z}} \Xi\left(M_{E}\right)_{\mathbb{Z}}=\Xi\left(M_{E}\right)$ and such that for all primes $p$ one has

$$
\vartheta_{p}\left(M_{E}, \Sigma\right)\left(\mathbb{Z}_{p} \otimes_{\mathbb{Z}} \Xi\left(M_{E}\right)_{\mathbb{Z}}\right)=\left[R \Gamma_{c, \text { ét }}\left(\mathcal{O}_{E, \Sigma_{p}}, T_{p}\right)\right]_{\mathbb{Z}_{p}}
$$

(cf. [6, Lem. 6]). Under this hypothesis we set

$$
\chi^{\mathrm{BK}}\left(M_{E}\right):=\vartheta_{\infty}\left(M_{E}\right)\left(\Xi\left(M_{E}\right)_{\mathbb{Z}}\right) \subset \mathbf{1}_{\mathbb{R}} .
$$

Then, after identifying $\mathbf{1}_{\mathbb{R}}$ with the graded module $(\mathbb{R}, 0)$ (as in Section A.1), the Tamagawa Number Conjecture of Bloch and Kato [4], as reformulated and extended by Fontaine and Perrin-Riou in [13], is equivalent to the following equality of sublattices of $\mathbb{R}$

$$
L^{*}\left(M_{E}, 0\right)^{-1} \cdot \mathbb{Z}=\chi^{\mathrm{BK}}\left(M_{E}\right),
$$

where $L^{*}\left(M_{E}, 0\right)$ denotes the leading term at $z=0$ of the (complex) $L$-function of the motive $M_{E}$ defined over $E$.

Remark 2.1. To define the virtual object $\Xi(M, E / k)$ and morphism $t_{j}^{\mathrm{BK}}(M, S, E / k)$ that occur above one must assume both the validity of the equivariant DeligneBeilinson Conjecture (as in [6, Conj. 1]) and the existence of equivariant Chern class maps (as in [6, Conj. 2]) for the motive $h^{0}(\operatorname{Spec}(E))(0) \otimes_{h^{0}(\operatorname{Spec}(k))(0)} M$, regarded as defined over $k$ and with coefficients $\mathbb{Q}\left[G_{E / k}\right]$.

\section{Main result}

3.1. Statement of the main result. The following result is the natural motivic generalization of a result that played a key role in the proof by Dokchitser and Dokchitser in [10] of the Parity Conjecture for Selmer ranks over $\mathbb{Q}$ (for more details of this connection see Section 4.1). Taken together with the standard properties of motivic $L$-functions under induction and inflation it shows that the conjectural equality (2.2) is compatible with Artin formalism.

For each subgroup $\Delta$ of a finite group $\Gamma$ and each commutative ring $R$ we write $R[\Gamma / \Delta]$ for the free $R$-module on the set $\{\Delta \gamma: \gamma \in \Gamma\}$ of right cosets of $\Delta$ in $\Gamma$. We regard $R[\Gamma / \Delta]$ as a (right) $R[\Gamma]$-module via the obvious right multiplication action of $\Gamma$ on $\{\Delta \gamma: \gamma \in \Gamma\}$. 
Theorem 3.1. Let $K / k$ be a finite Galois extension of number fields and set $G:=$ $G_{K / k}$. Let $M$ be a motive defined over $k$ and for each Galois extension $F / E$ with $k \subseteq$ $E \subseteq F \subseteq K$ assume the validity of both of the conjectures discussed in Remark 2.1 for the motive $h^{0}(\operatorname{Spec}(F)) \otimes_{h^{0}(\operatorname{Spec}(k))} M$, regarded as defined over $E$ and with coefficients $\mathbb{Q}\left[G_{F / E}\right]$.

Let $\left\{H_{a}: a \in A\right\}$ and $\left\{H_{b}^{\prime}: b \in B\right\}$ be finite sets of subgroups of $G$ such that the right $\mathbb{C}[G]$-modules $\bigoplus_{a \in A} \mathbb{C}\left[G / H_{a}\right]$ and $\bigoplus_{b \in B} \mathbb{C}\left[G / H_{b}^{\prime}\right]$ are isomorphic. Then for every prime $p$ and every isomorphism $j: \mathbb{C} \cong \mathbb{C}_{p}$ one has an equality

$$
\prod_{a \in A} \chi_{j}^{\mathrm{BK}}\left(M_{K^{H_{a}}}\right)=\prod_{b \in B} \chi_{j}^{\mathrm{BK}}\left(M_{K^{H_{b}^{\prime}}}\right)
$$

in $K_{0}\left(\mathbb{Z}_{p}, \mathbb{C}_{p}\right) \cong \mathbb{C}_{p}^{\times} / \mathbb{Z}_{p}^{\times}$

In particular, if in addition the Coherence hypothesis of [6, Section 3.3] is valid for the pair $\left(M_{K}, \mathbb{Q}[G]\right)$, then in $\mathbb{R}$ one has an equality of (free rank one) $\mathbb{Z}$-lattices

$$
\prod_{a \in A} \chi^{\mathrm{BK}}\left(M_{K^{H_{a}}}\right)=\prod_{b \in B} \chi^{\mathrm{BK}}\left(M_{K^{H_{b}^{\prime}}}\right) .
$$

Remark 3.2. Fix a number field $C$ with ring of integers $\mathcal{O}$ and set $C_{\mathbb{R}}:=\mathbb{R} \otimes_{\mathbb{Q}} C$. If $M$ has coefficients in $C$, then each motive $M_{E}$ has coefficients in $C$ and the construction of [6] gives, modulo the relevant cases of the conjectures discussed in Remark 2.1 and of the Coherence hypothesis of [6, Section 3.3], an object $\Xi\left(M_{E}\right)_{\mathcal{O}}$ of $V(\mathcal{O})$ and a canonical morphism $\mathbb{R} \otimes_{\mathbb{Z}} \Xi\left(M_{E}\right)_{\mathcal{O}} \rightarrow \mathbf{1}_{C_{\mathbb{R}}}$ in $V\left(C_{\mathbb{R}}\right)$. The leading term at $z=0$ of the $C$-equivariant (complex) $L$-function of $M_{E}$ is a unit of $C_{\mathbb{R}}$ and the analogue of the conjectural equality $(2.2)$ is an equality of invertible $\mathcal{O}$-submodules of $C_{\mathbb{R}}$. The result of Theorem 3.1 (and the proof presented below) extends directly to this setting but, for clarity of exposition, we prefer to leave all further details in this regard to the reader.

For a discussion of several applications of Theorem 3.1, see Section 4.

3.2. Proof of the main result. For any finitely generated module $Y$ we shall in the sequel write $Y_{p}$ and $Y_{\mathbb{C}_{p}}$ for $\mathbb{Z}_{p} \otimes_{\mathbb{Z}} Y$ and $\mathbb{C}_{p} \otimes_{\mathbb{Z}} Y$ respectively.

Before starting the proof of Theorem 3.1 we record a useful preliminary result.

Lemma 3.3. As $p$ runs over all primes and $j$ over all field isomorphisms $\mathbb{C} \cong \mathbb{C}_{p}$ the natural diagonal homomorphism $\mathbb{R}^{\times} / \mathbb{Z}^{\times} \rightarrow \prod_{p, j} \mathbb{C}_{p}^{\times} / \mathbb{Z}_{p}^{\times}$is injective.

Proof. Fix $x \in \mathbb{R}^{\times}$with $j(x) \in \mathbb{Z}_{p}^{\times} \subseteq \mathbb{C}_{p}^{\times}$for all $p$ and $j$. If $x$ was transcendental over $\mathbb{Q}$, then there would exist an isomorphism $j$ with $j(x) \notin \mathbb{Q}_{p}$ and so our assumptions imply that $x$ is algebraic over $\mathbb{Q}$. The fact that $j(x)$ belongs to $\mathbb{Q}_{p}$ for all $p$ and $j$ then also implies that all primes are completely split in the number field $\mathbb{Q}(x)$ generated by $x$ over $\mathbb{Q}$ so that $\mathbb{Q}(x)=\mathbb{Q}$ and hence $x$ is rational. Since $j(x)$ belongs to $\mathbb{Z}_{p}^{\times}$for all $p$ and $j$ this then implies that $x$ belongs to $\mathbb{Z}^{\times}$, as required.

To start the proof of Theorem 3.1, we note that if the Coherence hypothesis of $\left[6\right.$, Section 3.3] is valid for the pair $\left(M_{K}, \mathbb{Q}[G]\right)$, then it is clearly also valid for the pair $\left(M_{E}, \mathbb{Q}\right)$ for every intermediate field $E$ of $K / k$ and so all of the lattices $\chi^{\mathrm{BK}}\left(M_{E}\right)$ that occur in Theorem 3.1 are well defined. In addition, the equality (2.1) combines with the definitions of $\chi^{\mathrm{BK}}\left(M_{E}\right)$ and $\chi_{j}^{\mathrm{BK}}\left(M_{E}\right)$ to imply $\mathbb{Z}_{p} \otimes_{\mathbb{Z}} j\left(\chi^{\mathrm{BK}}\left(M_{E}\right)\right)=\chi_{j}^{\mathrm{BK}}\left(M_{E}\right)$. 
The latter equality then combines with the result of Lemma 3.3 to imply that the $\mathbb{Z}$-lattice $\chi^{\mathrm{BK}}\left(M_{E}\right)$ is uniquely determined by the $\mathbb{Z}_{p}$-lattices $\chi_{j}^{\mathrm{BK}}\left(M_{E}\right)$ for all primes $p$ and all isomorphisms $j: \mathbb{C} \cong \mathbb{C}_{p}$.

This observation shows, in particular, that the equality (3.2) is a consequence of the validity of (3.1) for all primes $p$ and all isomorphisms $j: \mathbb{C} \cong \mathbb{C}_{p}$. In the sequel it thus suffices to fix a prime $p$ and an isomorphism $j: \mathbb{C} \cong \mathbb{C}_{p}$ and then to prove the equality (3.1). To do this we adapt the approach used by Bartel in [1].

We thus define (right) $G$-modules by setting

$$
\Pi:=\bigoplus_{a \in A} \mathbb{Z}\left[G / H_{a}\right] \quad \text { and } \quad \Pi^{\prime}:=\bigoplus_{b \in B} \mathbb{Z}\left[G / H_{b}^{\prime}\right]
$$

and note the assumption that $\mathbb{C} \otimes_{\mathbb{Z}} \Pi$ and $\mathbb{C} \otimes_{\mathbb{Z}} \Pi^{\prime}$ are isomorphic $\mathbb{C}[G]$-modules combines with Deuring's theorem to imply the existence of a short exact sequence of $G$-modules

$$
0 \rightarrow \Pi \stackrel{\varphi}{\longrightarrow} \Pi^{\prime} \rightarrow \operatorname{cok}(\varphi) \rightarrow 0
$$

in which $\operatorname{cok}(\varphi)$ is finite. For any object $C$ of $D^{\mathrm{p}}\left(\mathbb{Z}_{p}[G]\right)$, the image under $\mathbb{Z}_{p} \otimes_{\mathbb{Z}}-$ of this exact sequence induces an exact triangle in $D^{\mathrm{p}}\left(\mathbb{Z}_{p}\right)$ of the form

$$
\Pi_{p} \otimes_{\mathbb{Z}_{p}[G]}^{\mathbb{L}} C \stackrel{\varphi_{p} \otimes_{\mathbb{Z}_{p}[G]}^{\mathbb{L}} \mathrm{id}_{C}}{\longrightarrow} \Pi_{p}^{\prime} \otimes_{\mathbb{Z}_{p}[G]}^{\mathbb{L}} C \rightarrow \operatorname{cok}(\varphi)_{p} \otimes_{\mathbb{Z}_{p}[G]}^{\mathbb{L}} C \rightarrow\left(\Pi_{p} \otimes_{\mathbb{Z}_{p}[G]}^{\mathbb{L}} C\right)[1]
$$

To study such triangles, we shall use the following technical result. To state this result we note that if $F$ is any field and $W$ any finitely generated right $F[G]$-module, then the assignment $P \mapsto P_{W}:=W \otimes_{F[G]} P$ (for each finitely generated $F[G]$-module $P$ ) induces a functor $V(F[G]) \rightarrow V(F)$. We write $t_{W}$ for the image of a morphism $t$ in $V(F[G])$ under this functor.

Lemma 3.4. Let $C$ be any object of $D^{\mathrm{p}}\left(\mathbb{Z}_{p}[G]\right)$. Then in each degree $m$ the module $H^{m}\left(\operatorname{cok}(\varphi)_{p} \otimes_{\mathbb{Z}_{p}[G]}^{\mathbb{L}} C\right)$ is finite. Further, if $t$ is any morphism in $V\left(\mathbb{C}_{p}[G]\right)$ of the form

$$
\left[\mathbb{C}_{p}[G] \otimes_{\mathbb{Z}_{p}[G]}^{\mathbb{L}} C\right]_{\mathbb{C}_{p}[G]} \rightarrow \prod_{m \in \mathbb{Z}}\left[H^{m}\left(\mathbb{C}_{p}[G] \otimes_{\mathbb{Z}_{p}[G]}^{\mathbb{L}} C\right)\right]_{\mathbb{C}_{p}[G]}^{(-1)^{m}} \rightarrow \mathbf{1}_{\mathbb{C}_{p}[G]}
$$

where the first arrow is the canonical morphism and the second is induced by a set of exact sequences of $\mathbb{C}_{p}[G]$-modules (in the sense of Section A.3), then in the group $K_{0}\left(\mathbb{Z}_{p}, \mathbb{C}_{p}\right) \cong \mathbb{C}_{p}^{\times} / \mathbb{Z}_{p}^{\times}$one has

$$
\begin{aligned}
& \chi^{\mathrm{ref}}\left(\Pi_{p}^{\prime} \otimes_{\mathbb{Z}_{p}[G]}^{\mathbb{L}} C, t_{\Pi_{\mathbb{C}_{p}}^{\prime}}\right) \\
& \quad=\chi^{\mathrm{ref}}\left(\Pi_{p} \otimes_{\mathbb{Z}_{p}[G]}^{\mathbb{L}} C, t_{\Pi_{\mathbb{C}_{p}}}\right) \cdot \prod_{m \in \mathbb{Z}}\left|H^{m}\left(\operatorname{cok}(\varphi)_{p} \otimes_{\mathbb{Z}_{p}[G]}^{\mathbb{L}} C\right)\right|^{(-1)^{m+1}} .
\end{aligned}
$$

Proof. Set $C_{\varphi}:=\operatorname{cok}(\varphi)_{p} \otimes_{\mathbb{Z}_{p}[G]}^{\mathbb{L}} C$. Then, since $C$ belongs to $D^{\mathrm{p}}\left(\mathbb{Z}_{p}[G]\right)$ and $\operatorname{cok}(\varphi)_{p}$ is finite, it is clear that each $\mathbb{Z}_{p}$-module $H^{m}\left(C_{\varphi}\right)$ is both finitely generated and torsion, and hence finite. 
We next apply Lemma A.1 with $\mathfrak{N}:=\left\{n \in \mathbb{Z}: H^{n}\left(\mathbb{C}_{p}[G] \otimes_{\mathbb{Z}_{p}[G]}^{\mathbb{L}} C\right) \neq 0\right\}, M_{n}:=$ $H^{n}\left(\mathbb{C}_{p}[G] \otimes_{\mathbb{Z}_{p}[G]}^{\mathbb{L}} C\right)$ for each $n \in \mathfrak{N}$ and $\phi:=\mathbb{C}_{p} \otimes_{\mathbb{Z}} \varphi$ to obtain a commutative diagram in $V\left(\mathbb{C}_{p}\right)$ of the form

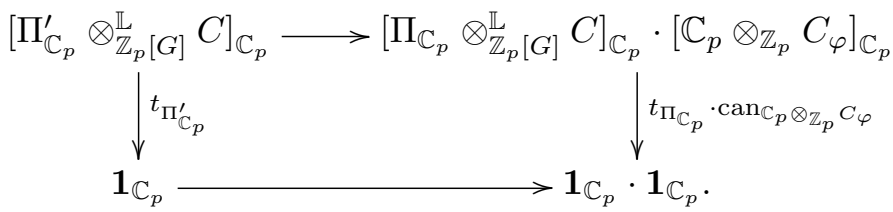

Here, the top arrow is the morphism induced by the image under $\mathbb{C}_{p}[G] \otimes_{\mathbb{Z}_{p}[G]}-$ of the exact triangle (3.4), the bottom arrow is the canonical morphism and 'can $\mathbb{C}_{p} \otimes_{\mathbb{Z}_{p}} C_{\varphi}$ ' denotes the morphism $\left[\mathbb{C}_{p} \otimes_{\mathbb{Z}_{p}} C_{\varphi}\right]_{\mathbb{C}_{p}} \rightarrow[0]_{\mathbb{C}_{p}}=\mathbf{1}_{\mathbb{C}_{p}}$ that is induced by the acyclicity of $\mathbb{C}_{p} \otimes_{\mathbb{Z}_{p}} C_{\varphi}$ (which itself follows from the fact that each module $H^{m}\left(C_{\varphi}\right)$ is finite).

The above diagram combines with the exact triangle (3.4) and the explicit product structure of the group $\pi_{0}\left(V\left(\mathbb{Z}_{p}\right) \times_{V\left(\mathbb{C}_{p}\right)} \mathcal{P}_{0}\right) \cong K_{0}\left(\mathbb{Z}_{p}, \mathbb{C}_{p}\right) \cong \mathbb{C}_{p}^{\times} / \mathbb{Z}_{p}^{\times}$to give an equality

$$
\chi^{\mathrm{ref}}\left(\Pi_{p}^{\prime} \otimes_{\mathbb{Z}_{p}[G]}^{\mathbb{L}} C, t_{\Pi_{\mathbb{C}_{p}}^{\prime}}\right)=\chi^{\mathrm{ref}}\left(\Pi_{p} \otimes_{\mathbb{Z}_{p}[G]}^{\mathbb{L}} C, t_{\Pi_{\mathbb{C}_{p}}}\right) \cdot \chi^{\mathrm{ref}}\left(C_{\varphi}, \operatorname{can}_{\mathbb{C}_{p} \otimes_{\mathbb{Z}_{p}} C_{\varphi}}\right) .
$$

This implies the equality (3.5) since the observation recalled in Section A.2 (with $R=$ $\mathbb{Z}_{p}, F=\mathbb{C}_{p}$ and $C=C_{\varphi}$ so $C_{F}$ is acyclic and hence $t_{\lambda}=\operatorname{can}_{\mathbb{C}_{p} \otimes_{\mathbb{Z}_{p}} C_{\varphi}}$ and $\left.\operatorname{det}(\lambda)=1\right)$ shows that $\chi^{\text {ref }}\left(C_{\varphi}, \operatorname{can}_{\mathbb{C}_{p} \otimes_{\mathbb{Z}_{p}} C_{\varphi}}\right)$ is equal to the image of $\prod_{m \in \mathbb{Z}}\left|H^{m}\left(C_{\varphi}\right)\right|^{(-1)^{m+1}}$ in $\mathbb{C}_{p}^{\times} / \mathbb{Z}_{p}^{\times}$

Returning to the proof of Theorem 3.1 we now set $C:=R \Gamma_{c \text {,ét }}\left(\mathcal{O}_{k, S_{p}}, T_{p, K}\right)$ and $t^{\mathrm{BK}}:=t_{j}^{\mathrm{BK}}(M, S, K / k)$. The definition of $t^{\mathrm{BK}}$ in [6] implies that it is induced by a finite set of exact sequences of $\mathbb{C}_{p}[G]$-modules in the sense of Section A.3 (with, in terms of the notation and numbering of [6], the necessary exact sequences being derived from (16), (17), (19), (22), (23), the central column of (26), (27), (28) and the isomorphism on cohomology induced by the quasi-isomorphism $\mathrm{AV}_{f}$ ).

By applying Lemma 3.4 with our current choice of $C$ and with $t=t^{\mathrm{BK}}$, we therefore deduce that

$$
\begin{aligned}
& \chi^{\mathrm{ref}}\left(\Pi_{p}^{\prime} \otimes_{\mathbb{Z}_{p}[G]}^{\mathbb{L}} C, t_{\Pi_{\mathbb{C}_{p}}^{\prime}}^{\mathrm{BK}}\right) \\
& \quad=\chi^{\mathrm{ref}}\left(\Pi_{p} \otimes_{\mathbb{Z}_{p}[G]}^{\mathbb{L}} C, t_{\Pi_{\mathbb{C}_{p}}}^{\mathrm{BK}}\right) \cdot \prod_{m \in \mathbb{Z}}\left|H^{m}\left(\operatorname{cok}(\varphi)_{p} \otimes_{\mathbb{Z}_{p}[G]}^{\mathbb{L}} C\right)\right|^{(-1)^{m+1}} .
\end{aligned}
$$

We note next that the identification $\Pi_{p} \otimes_{\mathbb{Z}_{p}[G]}^{\mathbb{L}} C=\bigoplus_{a \in A}\left(\mathbb{Z}_{p}\left[G / H_{a}\right] \otimes_{\mathbb{Z}_{p}[G]}^{\mathbb{L}} C\right)$ gives an equality in $K_{0}\left(\mathbb{Z}_{p}, \mathbb{C}_{p}\right)$

$$
\chi^{\mathrm{ref}}\left(\Pi_{p} \otimes_{\mathbb{Z}_{p}[G]}^{\mathbb{L}} C, t_{\Pi_{\mathbb{C}_{p}}}^{\mathrm{BK}}\right)=\prod_{a \in A} \chi^{\mathrm{ref}}\left(\mathbb{Z}_{p}\left[G / H_{a}\right] \otimes_{\mathbb{Z}_{p}[G]}^{\mathbb{L}} C, t_{\mathbb{C}_{p}\left[G / H_{a}\right]}^{\mathrm{BK}}\right),
$$

and similarly with $\Pi$ replaced by $\Pi^{\prime}$ and $\left\{H_{a}\right\}_{a \in A}$ by $\left\{H_{b}^{\prime}\right\}_{b \in B}$.

To compute the individual terms in these products we use the following result.

Lemma 3.5. Let $C$ and $t^{\mathrm{BK}}$ be fixed as above. Then for any subgroup $I$ of $G$ one has $\chi^{\mathrm{ref}}\left(\mathbb{Z}_{p}[G / I] \otimes_{\mathbb{Z}_{p}[G]}^{\mathbb{L}} C, t_{\mathbb{C}_{p}[G / I]}^{\mathrm{BK}}\right)=\chi_{j}^{\mathrm{BK}}\left(M_{K^{I}}\right)$ in $K_{0}\left(\mathbb{Z}_{p}, \mathbb{C}_{p}\right)$. 
Proof. For each subgroup $I$ of $G$ there is a natural composite isomorphism in $D^{\mathrm{p}}\left(\mathbb{Z}_{p}\right)$ of the form

$$
\begin{aligned}
\iota_{I} & : \mathbb{Z}_{p}[G / I] \otimes_{\mathbb{Z}_{p}[G]}^{\mathbb{L}} R \Gamma_{c, \text { ét }}\left(\mathcal{O}_{k, S_{p}}, T_{p, K}\right) \\
& \cong \mathbb{Z}_{p} \otimes_{\mathbb{Z}_{p}[I]}^{\mathbb{L}} R \Gamma_{c, \text { ét }}\left(\mathcal{O}_{k, S_{p}}, T_{p, K}\right) \\
& \cong R \Gamma_{c, \text { ét }}\left(\mathcal{O}_{k, S_{p}}, T_{p, K^{I}} \cong R \Gamma_{c, \text { ét }}\left(\mathcal{O}_{K^{I}, S_{p, K^{I}}}, T_{p}\right) .\right.
\end{aligned}
$$

The second isomorphism here is the standard descent isomorphism, the third is induced by Shapiro's Lemma and the first arises in the following way: for each finitely generated projective $\mathbb{Z}_{p}[G]$-module $P$ one has a composite isomorphism of $\mathbb{Z}_{p}$-modules

$$
\mathbb{Z}_{p}[G / I] \otimes_{\mathbb{Z}_{p}[G]} P=\{I\} \cdot \mathbb{Z}_{p}[G] \otimes_{\mathbb{Z}_{p}[G]} P \cong\{I\} \cdot \mathbb{Z}_{p} \otimes_{\mathbb{Z}_{p}[I]} P^{\prime} \cong \mathbb{Z}_{p} \otimes_{\mathbb{Z}_{p}[I]} P^{\prime},
$$

where $\{I\}$ denotes $I$ regarded as an element of $\mathbb{Z}_{p}[G / I], P^{\prime}$ denotes $P$ regarded, by restriction of scalars, as a $\mathbb{Z}_{p}[I]$-module and for each $\pi$ in $P^{\prime}$ the last map sends $\{I\} \otimes_{\mathbb{Z}_{p}[I]} \pi$ to $1 \otimes_{\mathbb{Z}_{p}[I]} \pi$.

Further, the argument of [6, Prop. 4.1] shows that the above isomorphism $\iota_{I}$ combines with the definitions of the morphisms $t^{\mathrm{BK}}:=t_{j}^{\mathrm{BK}}(M, S, K / k)$ and $t_{I}^{\mathrm{BK}}:=$ $t_{j}^{\mathrm{BK}}\left(M_{K^{I}}, S_{K^{I}}\right)$ to give a commutative diagram in $V\left(\mathbb{C}_{p}\right)$

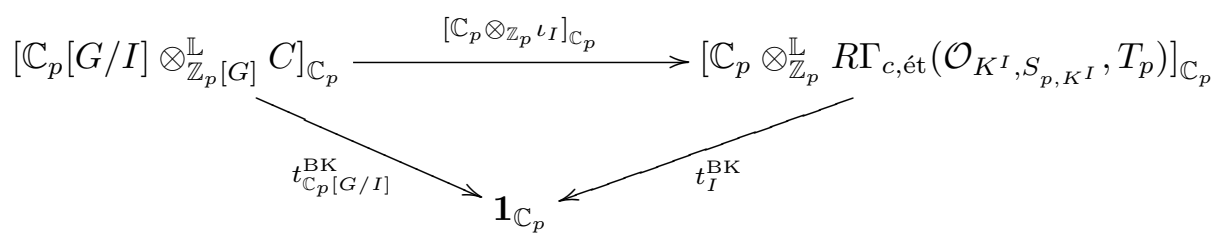

The pair $\left(\iota_{I},\left[\mathbb{C}_{p} \otimes_{\mathbb{Z}_{p}} \iota_{I}\right]_{\mathbb{C}_{p}}\right)$ therefore constitutes an isomorphism in $V\left(\mathbb{Z}_{p}\right) \times_{V\left(\mathbb{C}_{p}\right)} \mathcal{P}_{0}$ between $\left(\left[\mathbb{Z}_{p}[G / I] \otimes_{\mathbb{Z}_{p}[G]}^{\mathbb{L}} C\right]_{\mathbb{Z}_{p}}, t_{\mathbb{C}_{p}[G / I]}^{\mathrm{BK}}\right)$ and $\left(\left[R \Gamma_{c \text {,ét }}\left(\mathcal{O}_{K^{I}, S_{p, K^{I}}}, T_{p}\right)\right]_{\mathbb{Z}_{p}}, t_{I}^{\mathrm{BK}}\right)$ and hence implies that in $\pi_{0}\left(V\left(\mathbb{Z}_{p}\right) \times_{V\left(\mathbb{C}_{p}\right)} \mathcal{P}_{0}\right) \cong K_{0}\left(\mathbb{Z}_{p}, \mathbb{C}_{p}\right)$, one has an equality

$$
\chi^{\mathrm{ref}}\left(\mathbb{Z}_{p}[G / I] \otimes_{\mathbb{Z}_{p}[G]}^{\mathbb{L}} C, t_{\mathbb{C}_{p}[G / I]}^{\mathrm{BK}}\right)=\chi^{\mathrm{ref}}\left(R \Gamma_{c, \text { ét }}\left(\mathcal{O}_{K^{I}, S_{p, K^{I}}}, T_{p}\right), t_{I}^{\mathrm{BK}}\right)=: \chi_{j}^{\mathrm{BK}}\left(M_{K^{I}}\right),
$$

as required.

Upon combining Lemma 3.5 with the equalities (3.6) and (3.7) (and the analogous equality for $\Pi^{\prime}$ ), one obtains an equality

$$
\prod_{b \in B} \chi_{j}^{\mathrm{BK}}\left(M_{K^{H_{b}^{\prime}}}\right)=\prod_{a \in A} \chi_{j}^{\mathrm{BK}}\left(M_{K^{H_{a}}}\right) \cdot \prod_{m \in \mathbb{Z}}\left|H^{m}\left(\operatorname{cok}(\varphi)_{p} \otimes_{\mathbb{Z}_{p}[G]}^{\mathbb{L}} C\right)\right|^{(-1)^{m+1}} .
$$

The proof of Theorem 3.1 is therefore completed by the following result.

Lemma 3.6. $\prod_{m \in \mathbb{Z}}\left|H^{m}\left(\operatorname{cok}(\varphi)_{p} \otimes_{\mathbb{Z}_{p}[G]}^{\mathbb{L}} C\right)\right|^{(-1)^{m}}=1$.

Proof. For each $\mathbb{Z}_{p}$-module $M$ and natural number $n$ we write $M^{(n)}$ for the direct sum of $n$ copies of $M$. 
We first claim that there are non-negative integers $\left\{n_{m}: m \in \mathbb{Z}\right\}$, with $n_{m}=0$ for almost all $m$, such that $C$ is represented by a (bounded) complex of the form

$$
P^{\bullet}=\left[\cdots \rightarrow \mathbb{Z}_{p}[G]^{\left(n_{m}\right)} \rightarrow \mathbb{Z}_{p}[G]^{\left(n_{m+1}\right)} \rightarrow \cdots\right],
$$

where the term $\mathbb{Z}_{p}[G]^{\left(n_{m}\right)}$ occurs in degree $m$ and one has $\sum_{m \in \mathbb{Z}}(-1)^{m} n_{m}=0$. Indeed, since $C$ belongs to $D^{\mathrm{p}}\left(\mathbb{Z}_{p}[G]\right)$ and the Krull-Schimdt-Azumaya Theorem applies to the algebra $\mathbb{Z}_{p}[G]$, to prove that $C$ is isomorphic in $D^{\mathrm{p}}\left(\mathbb{Z}_{p}[G]\right)$ to a complex of the form $P^{\bullet}$ it suffices to show that the Euler characteristic of $C$ in $K_{0}\left(\mathbb{Z}_{p}[G]\right)$ vanishes. We set $\mathbb{F}=\mathbb{Z} / p \mathbb{Z}$. Then the natural reduction map $K_{0}\left(\mathbb{Z}_{p}[G]\right) \rightarrow K_{0}(\mathbb{F}[G])$ is bijective (cf. [3, Chap. IX, Prop. 1.3]) and so it is enough to show that the Euler characteristic of $\mathbb{F}[G] \otimes_{\mathbb{Z}_{p}[G]}^{\mathbb{L}} C$ in $K_{0}(\mathbb{F}[G])$ vanishes. But $\mathbb{F}[G] \otimes_{\mathbb{Z}_{p}[G]}^{\mathbb{L}} C$ is naturally isomorphic in $D^{\mathrm{p}}(\mathbb{F}[G])$ to $R \Gamma_{c \text {,ét }}\left(\mathcal{O}_{k, S_{p}}, T_{p} \otimes_{\mathbb{Z}_{p}} \prod_{K \rightarrow k^{c}} \mathbb{F}\right)$ and the Euler characteristic in $K_{0}(\mathbb{F}[G])$ of the latter complex vanishes as a direct consequence of Flach's equivariant refinement of Tate's formula for the global Euler characteristic [12, Th. 5.1].

Replacing $C$ by $P^{\bullet}$ shows that the derived tensor product $\operatorname{cok}(\varphi)_{p} \otimes_{\mathbb{Z}_{p}[G]}^{\mathbb{L}} C$ is represented by the bounded complex

$$
\operatorname{cok}(\varphi)_{p} \otimes_{\mathbb{Z}_{p}[G]} P^{\bullet}=\left[\cdots \rightarrow \operatorname{cok}(\varphi)_{p}^{\left(n_{m}\right)} \rightarrow \operatorname{cok}(\varphi)_{p}^{\left(n_{m+1}\right)} \rightarrow \cdots\right],
$$

where each module $\operatorname{cok}(\varphi)_{p}^{\left(n_{m}\right)}$ occurs in degree $m$. By breaking this complex into the associated short exact sequences of boundaries, cycles and cohomology, and noting that all such groups are finite, one then computes that

$$
\begin{aligned}
\prod_{m \in \mathbb{Z}}\left|H^{m}\left(\operatorname{cok}(\varphi)_{p} \otimes_{\mathbb{Z}_{p}[G]}^{\mathbb{L}} C\right)\right|^{(-1)^{m}} & =\prod_{m \in \mathbb{Z}}\left|H^{m}\left(\operatorname{cok}(\varphi)_{p} \otimes_{\mathbb{Z}_{p}[G]} P^{\bullet}\right)\right|^{(-1)^{m}} \\
& =\prod_{m \in \mathbb{Z}}\left|\operatorname{cok}(\varphi)_{p}^{\left(n_{m}\right)}\right|^{(-1)^{m}} \\
& =\prod_{m \in \mathbb{Z}}\left|\operatorname{cok}(\varphi)_{p}\right|^{(-1)^{m} n_{m}} \\
& =\left|\operatorname{cok}(\varphi)_{p}\right|^{\sum_{m \in \mathbb{Z}}(-1)^{m} n_{m}} \\
& =\left|\operatorname{cok}(\varphi)_{p}\right|^{0}
\end{aligned}
$$

which is equal to 1 , as required.

\section{Applications}

In this section, we discuss three explicit applications of Theorem 3.1 and then describe an alternative approach to the theory of regulator constants introduced by Dokchitser and Dokchitser.

In our first two applications, we merely obtain a more conceptual proof of results that are already in the literature and so give only brief details but our final application is a new result that has since been applied elsewhere by other authors and so we give a detailed argument.

We fix a finite Galois extension of number fields $K / k$ and a field $E$ with $k \subseteq E \subseteq K$. 
4.1. Abelian varieties. Let $X$ be an abelian variety that is defined over $k$ and set $M:=h^{1}(X)(1)$.

In this case, all of the hypotheses in the first paragraph of Theorem 3.1 are known to be satisfied and, in addition, if the (classical) Tate-Shafarevic group of $X$ over $K$ is finite, then the pair $\left(M_{K}, \mathbb{Q}[G]\right)$ satisfies the 'Coherence hypothesis' of [6, Section 3.3] and so Theorem 3.1 is unconditional. In the latter case, one can also show that $\chi^{\mathrm{BK}}\left(M_{E}\right)$ is equal to the $\mathbb{Z}$-submodule of $\mathbb{R}$ that is generated by the inverse of the 'BSD quotient' $\operatorname{BSD}(X / E)$ defined by Dokchitser and Dokchitser in [10, Section 2.1] (this fact follows, for example, from the explicit computation of Venjakob in [18, Section 3.1]).

In this way Theorem 3.1 gives an alternative proof of the result of [10, Th. 2.3] that played a key role in the proof of the Parity Conjecture for Selmer ranks over $\mathbb{Q}$ that is given in loc. cit.

4.2. Units and ideal class groups. We now set $M:=h^{0}(\operatorname{Spec}(k))(0)$.

In this case all of the hypotheses in Theorem 3.1 are satisfied and each sublattice $\chi^{\mathrm{BK}}\left(M_{E}\right)$ of $\mathbb{R}$ can be computed explicitly by using Kummer theory and class field theory. Further, the $L$-function $L\left(M_{E}, z\right)$ is equal to the Dedekind zeta function of $E$ and the equality (2.2) is known to be equivalent (modulo a sign) to the analytic class number formula for $E$.

A slight generalization of this result is also useful in applications and to quickly describe this we fix a prime $p$, an isomorphism $j: \mathbb{C} \cong \mathbb{C}_{p}$ and a finite set of places $\Sigma$ of $E$ with $S_{\infty, E} \subseteq \Sigma$. Then the proof of [5, Prop. 4.2.2] combines with the observation made in Section A.2 below to show the existence of a morphism

$$
t_{\Sigma, j}^{\mathrm{BK}}: \mathbb{C}_{p} \otimes_{\mathbb{Z}_{p}}\left[R \Gamma_{c, \text { ét }}\left(\mathcal{O}_{E, \Sigma_{p}}, \mathbb{Z}_{p}\right)\right]_{\mathbb{Z}_{p}} \rightarrow \mathbf{1}_{\mathbb{C}_{p}}
$$

with all of the following properties: $t_{\Sigma, j}^{\mathrm{BK}}=t_{j}^{\mathrm{BK}}\left(M_{E}, \Sigma\right)$ if $\Sigma=S_{\infty, E} ; t_{\Sigma, j}^{\mathrm{BK}}$ is induced by a set of exact sequences of $\mathbb{C}_{p}$-modules (in the sense of Section A.3); the element $\chi^{\mathrm{ref}}\left(R \Gamma_{c, \text { ét }}\left(\mathcal{O}_{E, \Sigma_{p}}, \mathbb{Z}_{p}\right), t_{\Sigma, j}^{\mathrm{BK}}\right)$ corresponds to the sublattice $\left(j\left(R_{E, \Sigma}\right) h_{E, \Sigma} / w_{E}\right)^{-1} \cdot \mathbb{Z}_{p}$ of $\mathbb{C}_{p}$ where $R_{E, \Sigma}$ is the $\Sigma$-regulator of $E$ and $h_{E, \Sigma}$ and $w_{E}$ are the orders of the groups $\mathrm{Cl}\left(\mathcal{O}_{E, \Sigma}\right)$ and $\left(E^{\times}\right)_{\text {tor }}$, respectively.

By using the morphisms $t_{S_{E}, j}^{\mathrm{BK}}$ for the various intermediate fields $E$ of $K / k$, and with $S$ any fixed finite set of places of $k$ containing $S_{\infty, k}$, Theorem 3.1 can be used to give an alternative proof of the equality that is proved by Bartel in $[1,(1)]$. We note that while this proof is ostensibly more involved than the proof given in loc. cit. it is also conceptually different in that it uses only Galois cohomology rather than analytic methods.

In particular, this approach shows that the results of Bartel's paper (and earlier results of, amongst others, de Smit [7]) are purely algebraic in nature and do not depend on the analytic class number formula.

4.3. Higher algebraic $\boldsymbol{K}$-groups. Recall that for each number field $E$, strictly negative integer $r$, integer $i \in\{1,2\}$ and odd prime $p$ Soulé [16] and Dwyer and Friedlander [11] have constructed a canonical Chern class homomorphism

$$
\operatorname{ch}_{E, p, r}^{i}: K_{2-i-2 r}\left(\mathcal{O}_{E}\right)_{p} \rightarrow H_{\text {ét }}^{i}\left(\mathcal{O}_{E}^{p}, \mathbb{Z}_{p}(1-r)\right)
$$

where we set $\mathcal{O}_{E}^{p}:=\mathcal{O}_{E}\left[\frac{1}{p}\right]$. 
The kernel of $\operatorname{ch}_{E, p, r}^{1}$ is known to be finite and we write $w_{r, E}$ and $h_{r, E}$ for the orders of the (finite) groups $W_{r, E}:=\bigoplus_{p \neq 2} K_{1-2 r}\left(\mathcal{O}_{E}\right)_{p, \text { tor }} / \operatorname{ker}\left(\operatorname{ch}_{E, p, r}^{1}\right)$ and $H_{r, E}:=$ $\bigoplus_{p \neq 2} K_{-2 r}\left(\mathcal{O}_{E}\right)_{p} / \operatorname{ker}\left(\operatorname{ch}_{E, p, r}^{2}\right)$, respectively. We also write $R_{r, E}$ for the Borel regulator in degree $1-2 r$ of the field $E$.

The following consequence of Theorem 3.1 is a natural analogue for higher algebraic $K$-groups of the equality recorded by Bartel in $[1,(1)]$. This result implies that Lichtenbaum's conjecture on the leading terms of Dedekind Zeta functions at strictly negative integers is compatible with Artin formalism and has also recently been used by Bartel and de Smit in [2] to prove some interesting new (and unconditional) results about the explicit Galois structure of higher algebraic $K$-groups.

Corollary 4.1. Let $K / k$ be a finite Galois extension of number fields and set $G:=$ $G_{K / k}$. Let $\left\{H_{a}: a \in A\right\}$ and $\left\{H_{b}^{\prime}: b \in B\right\}$ be finite sets of subgroups of $G$ such that the right $\mathbb{C}[G]$-modules $\bigoplus_{a \in A} \mathbb{C}\left[G / H_{a}\right]$ and $\bigoplus_{b \in B} \mathbb{C}\left[G / H_{b}^{\prime}\right]$ are isomorphic. Then for each strictly negative integer $r$ there exists an integer $m$ such that

$$
\prod_{a \in A}\left(h_{r, K^{H_{a}}} R_{r, K^{H_{a}}} / w_{r, K^{H_{a}}}\right)=2^{m} \prod_{b \in B}\left(h_{r, K^{H_{b}^{\prime}}} R_{r, K^{H_{b}^{\prime}}} / w_{r, K^{H_{b}^{\prime}}}\right) .
$$

Proof. Fix a strictly negative integer $r$ and set $M:=h^{0}(\operatorname{Spec}(k))(r)$. In this case, all of the hypotheses in Theorem 3.1 are satisfied and so, given the final assertion of that result, it is enough to prove that for each intermediate field $E$ of $K / k$ and each odd prime $p$ one has $\chi^{\mathrm{BK}}\left(M_{E}\right)_{p}=\left(h_{r, E} R_{r, E} / w_{r, E}\right)^{-1} \mathbb{Z}_{p}$.

To show this, we note that (since $p$ is odd) the Artin-Verdier Duality Theorem gives an exact triangle in $D^{\mathrm{p}}\left(\mathbb{Z}_{p}\right)$ of the form

$$
C_{c, E} \rightarrow R \operatorname{Hom}_{\mathbb{Z}_{p}}\left(C_{E}^{\prime}, \mathbb{Z}_{p}\right)[-3] \rightarrow B_{r, E}[0] \rightarrow C_{c, E}[1],
$$

where we set $C_{c, E}:=R \Gamma_{c \text {,ét }}\left(\mathcal{O}_{E}^{p}, \mathbb{Z}_{p}(r)\right), C_{E}^{\prime}:=R \Gamma_{\text {ét }}\left(\mathcal{O}_{E}^{p}, \mathbb{Z}_{p}(1-r)\right)$ and $B_{r, E}:=$ $\bigoplus_{w \in S_{\infty, E}} H_{\text {ét }}^{0}\left(L_{w}, \mathbb{Z}_{p}(r)\right)$. The complex $C_{E}^{\prime}$ is acyclic outside degrees one and two and the (surjective) $\operatorname{maps} \operatorname{ch}_{E, p, r}^{1}$ and $\operatorname{ch}_{E, p, r}^{2}$ induce isomorphisms $H^{1}\left(C_{E}^{\prime}\right) \cong W_{r, E}^{p}:=$ $K_{1-2 r}\left(\mathcal{O}_{E}\right)_{p} / \operatorname{ker}\left(\operatorname{ch}_{E, p, r}^{1}\right)$ and $H^{2}\left(C_{E}^{\prime}\right) \cong H_{r, E, p}$. It is now easy to derive explicit descriptions of the groups $H^{i}\left(C_{c, E}\right)$ by using the long exact cohomology sequence of (4.1): one finds that $H^{i}\left(C_{c, E}\right)=\{0\}$ if $i \notin\{1,2,3\}$ and that there are natural isomorphisms $H^{1}\left(C_{c, E}\right) \cong B_{r, E}$ and $H^{3}\left(C_{c, E}\right) \cong \operatorname{Hom}_{\mathbb{Z}}\left(W_{r, E}, \mathbb{Q}_{p} / \mathbb{Z}_{p}\right)$ and an exact sequence

$$
0 \rightarrow \operatorname{Hom}_{\mathbb{Z}}\left(H_{r, E}, \mathbb{Q}_{p} / \mathbb{Z}_{p}\right) \rightarrow H^{2}\left(C_{c, E}\right) \rightarrow \operatorname{Hom}_{\mathbb{Z}_{p}}\left(W_{r, E}^{p}, \mathbb{Z}_{p}\right) \rightarrow 0 .
$$

Further, with respect to these descriptions, one has $t_{j}^{\mathrm{BK}}\left(M_{E}, S_{\infty, E}\right)=t_{\operatorname{Hom}_{\mathbb{C}_{p}}\left(\beta, \mathbb{C}_{p}\right)}$ where $\beta$ is the isomorphism $\mathbb{C}_{p} \otimes_{\mathbb{Z}} K_{1-2 r}\left(\mathcal{O}_{E}\right) \cong \operatorname{Hom}_{\mathbb{Z}_{p}}\left(B_{r, E}, \mathbb{C}_{p}\right)$ that is induced by the Borel regulator map (and we have used the notation of Section A.2).

Given these facts, the required equality $\chi^{\mathrm{BK}}\left(M_{E}\right)_{p}=\left(h_{r, E} R_{r, E} / w_{r, E}\right)^{-1} \mathbb{Z}_{p}$ is a consequence of the formula (A.1) below (with $R=\mathbb{Z}_{p}, F=\mathbb{C}_{p}, C=C_{c, E}, a=1$ and $\left.\lambda=\operatorname{Hom}_{\mathbb{C}_{p}}\left(\beta, \mathbb{C}_{p}\right)\right)$.

Remark 4.2. Regarding the equality in Corollary 4.1 note that if the maps $\operatorname{ch}_{E, p, r}^{i}$ are injective, as has been conjectured by Quillen and Lichtenbaum, then $h_{r, E}=$ $\left|K_{-2 r}\left(\mathcal{O}_{E}\right)\left[\frac{1}{2}\right]\right|$ and $w_{r, E}=\left|K_{1-2 r}\left(\mathcal{O}_{E}\right)_{\text {tor }}\left[\frac{1}{2}\right]\right|$. If $r=-1$, then the injectivity of the maps $\mathrm{ch}_{E, p, r}^{i}$ follows from the work of Tate [17], Levine [14] and Merkuriev and Suslin 
[15]. In general it is known, by work of Suslin, that the injectivity of $\operatorname{ch}_{E, p, r}^{i}$ is a consequence of the conjecture of Bloch and Kato relating Milnor $K$-theory to étale cohomology and it is widely believed that recent work of Voevodsky and Rost has completed the proof of the Bloch-Kato Conjecture.

4.4. Regulator constants. In this subsection, we show that the techniques of the present paper also give a different approach to the theory of 'regulator constants' that was introduced by Dokchitser and Dokchitser in [10] and has played a key role in several subsequent articles including $[1,9]$.

To explain this point we fix a field $F$ of characteristic 0 and let $C$ denote the complex $I \stackrel{0}{\rightarrow} J$ where $I$ and $J$ are finitely generated $F[G]$-modules and $I$ is placed in degree zero. We also fix a finitely generated right $F[G]$-module $W$ and set $I_{W}:=W \otimes_{F[G]} I$, $J_{W}:=W \otimes_{F[G]} J$ and $C_{W}:=W \otimes_{F[G]} C$. We identify $V(F)$ with the category of graded line bundles on $F$ as in Section A.1 and set $Y^{*}:=\operatorname{Hom}_{F}(Y, F)$ for any $F$-module $Y$.

Then any isomorphism of $F[G]$-modules $\iota: I \stackrel{\sim}{\longrightarrow} J$ induces a morphism in $V(F[G])$ of the form $t_{\iota}:[C]_{F[G]} \rightarrow \mathbf{1}_{F[G]}$ (see Section A.2) and, setting $d:=\operatorname{dim}_{F}\left(I_{W}\right)=$ $\operatorname{dim}_{F}\left(J_{W}\right)$, the induced morphism $t_{\iota, W}:\left[C_{W}\right]_{F} \rightarrow \mathbf{1}_{F}$ in $V(F)$ sends each element $(x \otimes$ $f, 0)$ of $\left(\wedge_{F}^{d} I_{W} \otimes_{F}\left(\wedge_{F}^{d} J_{W}\right)^{*}, 0\right)=\left[C_{W}\right]_{F}$ to $\left(f\left(\wedge_{F}^{d}\left(\operatorname{id}_{W} \otimes_{F[G]} \iota\right)(x)\right), 0\right) \in(F, 0)=\mathbf{1}_{F}$.

In the following result, we write $\psi^{\text {tr }}$ for the transpose of a linear map $\psi$.

Lemma 4.3. Let $\phi: W \rightarrow W^{\prime}$ be an isomorphism of finitely generated right $F[G]$-modules and set $\phi_{I}:=\phi \otimes_{F[G]} \mathrm{id}_{I}$ and $\phi_{J}:=\phi \otimes_{F[G]} \mathrm{id}_{J}$. Then for any non-zero elements $x, y, x^{\prime}$ and $y^{\prime}$ of $\wedge_{F}^{d} I_{W},\left(\wedge_{F}^{d} J_{W}\right)^{*}, \wedge_{F}^{d} I_{W^{\prime}}$ and $\left(\wedge_{F}^{d} J_{W^{\prime}}\right)^{*}$, respectively, one has

$$
t_{\iota, W}(x \otimes y) t_{\iota, W^{\prime}}\left(x^{\prime} \otimes y^{\prime}\right)^{-1}=c(\phi)_{x, x^{\prime}} c\left(\phi^{\mathrm{tr}}\right)_{y^{\prime}, y}^{-1},
$$

where $c(\phi)_{x, x^{\prime}}$ and $c\left(\phi^{\text {tr }}\right)_{y^{\prime}, y}$ are the elements of $F^{\times}$defined by setting $\wedge_{F}^{d} \phi_{I}(x)=$ $c(\phi)_{x, x^{\prime}} \cdot x^{\prime}$ and $\left(\wedge_{F}^{d} \phi_{J}^{\mathrm{tr}}\right)\left(y^{\prime}\right)=c\left(\phi^{\mathrm{tr}}\right)_{y^{\prime}, y} \cdot y$. In particular, the expression in (4.2) is independent of both $\iota$ and $\phi$.

Proof. Setting $\iota_{W}:=\mathrm{id}_{W} \otimes_{F[G]} \iota$ and $\iota_{W^{\prime}}:=\mathrm{id}_{W^{\prime}} \otimes_{F[G]} \iota$ it is clear that $\phi_{J} \circ \iota_{W}=$ $\iota_{W} \circ \phi_{I}$. From the commutative diagram at the end of the proof of Lemma A.1 below with $N=J$ it therefore follows that $t_{\iota, W}(x \otimes y)$ is equal to

$$
\begin{aligned}
\mathrm{ev}_{J_{W}}\left(\wedge_{F}^{d} \iota_{W}(x) \otimes y\right) & =\operatorname{ev}_{J_{W^{\prime}}}\left(\wedge_{F}^{d} \phi_{J}\left(\wedge_{F}^{d} \iota_{W}(x)\right) \otimes\left(\wedge_{F} \phi_{J}^{\mathrm{tr}}\right)^{-1}(y)\right) \\
& =\operatorname{ev}_{J_{W^{\prime}}}\left(\wedge_{F}^{d} \iota_{W^{\prime}}\left(\wedge_{F}^{d} \phi_{I}(x)\right) \otimes\left(\wedge_{F} \phi_{J}^{\mathrm{tr}}\right)^{-1}(y)\right) \\
& =t_{\iota, W^{\prime}}\left(\wedge_{F}^{d} \phi_{I}(x) \otimes\left(\wedge_{F} \phi_{J}^{\mathrm{tr}}\right)^{-1}(y)\right) \\
& =t_{\iota, W^{\prime}}\left(c(\phi)_{x, x^{\prime}} \cdot x^{\prime} \otimes c\left(\phi^{\mathrm{tr}}\right)_{y^{\prime}, y}^{-1} \cdot y^{\prime}\right) \\
& =c(\phi)_{x, x^{\prime}} c\left(\phi^{\mathrm{tr}}\right)_{y^{\prime}, y}^{-1} \cdot t_{\iota, W^{\prime}}\left(x^{\prime} \otimes y^{\prime}\right),
\end{aligned}
$$

as required to prove the equality (4.2). The final assertion of the lemma is then true because the left- and right-hand sides of (4.2) are obviously independent of $\phi$ and $\iota$, respectively.

To explain the connection of Lemma 4.3 to the theory of regulator constants we consider the special case that $J=I^{*}$ and the $F[G]$-modules $W$ and $W^{\prime}$ are selfdual. Then each choice of isomorphisms of $F[G]$-modules $W \cong W^{*}$ and $W^{\prime} \cong W^{\prime *}$ 
induces identifications of $F$-modules $\left(J_{W}\right)^{*} \cong J_{W^{*}}^{*} \cong\left(I^{* *}\right)_{W} \cong I_{W}$ and similarly $\left(J_{W^{\prime}}\right)^{*} \cong I_{W^{\prime}}$ and hence also $\left(\wedge_{F}^{d} J_{W}\right)^{*} \cong \wedge_{F}^{d}\left(J_{W}\right)^{*} \cong \wedge_{F}^{d} I_{W}$ and $\left(\wedge_{F}^{d} J_{W^{\prime}}\right)^{*} \cong \wedge_{F}^{d} I_{W^{\prime}}$. Fixing such identifications allows us to choose $x=y$ and $x^{\prime}=y^{\prime}$ in Lemma 4.3 and in this case (4.2) implies immediately that the quotient $t_{\iota, W}(x \otimes x) t_{\iota, W^{\prime}}\left(x^{\prime} \otimes x^{\prime}\right)^{-1}$ is independent of the choices of $x$ and $x^{\prime}$ when considered as an element of $F^{\times} /\left(F^{\times}\right)^{2}$. In particular, if we now suppose that $F$ contains a Dedekind domain $R$, that $I=F \otimes_{R} M$ for an $R[G]$-lattice $M$, that the isomorphism of $F[G]$-modules $\iota: I \rightarrow I^{*}$ is induced by a non-degenerate $G$-invariant pairing $M \times M \rightarrow F$ and that $W$ and $W^{\prime}$ are the (canonically self-dual) $F[G]$-modules $F \otimes_{\mathbb{Z}} \Pi$ and $F \otimes_{\mathbb{Z}} \Pi^{\prime}$ defined in (3.3), then for a suitable choice of elements $x$ and $x^{\prime}$ the quotient $t_{\iota, W}(x \otimes x) t_{\iota, W^{\prime}}\left(x^{\prime} \otimes x^{\prime}\right)^{-1}$ is a 'regulator constant' as defined in [10].

In this way, Lemma 4.3 gives different proofs of the results in both [10, Section 2.3] and $[1$, Section 3$]$.

\section{Appendix A. Virtual objects and relative algebraic $K$-theory}

For the reader's convenience, we quickly recall some standard facts concerning virtual objects. Categories of virtual objects were first introduced by Deligne in [8] and a fuller review of their properties than is given here (in particular, of the connection to relative algebraic $K$-theory) can be found in [6, Section 2].

A.1. We fix an associative unital noetherian $\operatorname{ring} R$. Modules over $R$ are to be understood, unless explicitly stated otherwise, as left modules. We use the notation $V(R)$, $[M]_{R}, X \cdot Y, \mathbf{1}_{R}, X^{-1}, \mathrm{ev}_{X}$ and $D^{\mathrm{p}}(R)$ introduced in Section 2.1 .

Each object $C$ of $D^{\mathrm{p}}(R)$ gives rise to a canonical object $[C]_{R}$ of $V(R)$. If $R$ is regular, then for any such $C$ there is a canonical morphism in $V(R)$ of the form

$$
\iota(C):[C]_{R} \rightarrow \prod_{m \in \mathbb{Z}}\left[H^{m}(C)\right]_{R}^{(-1)^{m}} .
$$

If $R$ is a field, then one can identify $V(R)$ with the category of graded line bundles on $R$ in such a way that for any $R$-module $M$ of rank $r$ one has $[M]_{R}=$ $\left(\wedge_{R}^{r} M, r\right),[M]_{R}^{-1}=\left(\operatorname{Hom}_{R}\left(\wedge_{R}^{r} M, R\right),-r\right),[M]_{R} \cdot[N]_{R}=\left(\wedge_{R}^{r} M \otimes_{R} \wedge_{R}^{r^{\prime}} N, r+r^{\prime}\right)$ for any $R$-module $N$ of rank $r^{\prime}$, and ev $_{M}$ is induced by the natural evaluation pairing $\wedge_{R}^{r} M \otimes_{R} \operatorname{Hom}_{R}\left(\wedge_{R}^{r} M, R\right) \rightarrow R$.

A.2. We now let $R$ be a discrete valuation ring of characteristic 0 and $F$ a field that contains $R$. We fix an object $C$ of $D^{\mathrm{p}}(R)$ and set $C_{F}:=F \otimes_{R}^{\mathbb{L}} C$. For each morphism $t:\left[C_{F}\right]_{F} \rightarrow \mathbf{1}_{F}$ in $V(F)$, we recall the notation $\chi^{\mathrm{ref}}(C, t)$ introduced in Section 2.1. We also use the natural isomorphism $K_{0}(R, F) \cong F^{\times} / R^{\times}$to identify $\chi^{\text {ref }}(C, t)$ with a (free) rank one $R$-submodule of $F$. We recall the following special case of this construction.

If $C_{F}$ is acyclic outside degrees $a$ and $a+1$ (for any given integer $a$ ), then any isomorphism of $F$-modules $\lambda: H^{a}\left(C_{F}\right) \cong H^{a+1}\left(C_{F}\right)$ induces a composite morphism

$$
\begin{aligned}
t_{\lambda}: F \otimes_{R}[C]_{R} \rightarrow\left[C_{F}\right]_{F} \stackrel{\iota\left(C_{F}\right)}{\longrightarrow}\left[H^{a}\left(C_{F}\right)\right]_{F}^{(-1)^{a}} \cdot\left[H^{a+1}\left(C_{F}\right)\right]_{F}^{(-1)^{a+1}} \\
\quad \stackrel{[\lambda]_{F}^{(-1)^{a}} \cdot \mathrm{id}}{\longrightarrow}\left[H^{a+1}\left(C_{F}\right)\right]_{F}^{(-1)^{a}} \cdot\left[H^{a+1}\left(C_{F}\right)\right]_{F}^{(-1)^{a+1}} \stackrel{\operatorname{ev}_{\left[H^{a+1}\left(C_{F}\right)\right]_{F}^{(-1)^{a}}}^{\longrightarrow}}{\longrightarrow} \mathbf{1}_{F},
\end{aligned}
$$


and one has

$$
\chi^{\mathrm{ref}}\left(C, t_{\lambda}\right)=\operatorname{det}(\lambda)^{(-1)^{a}} \prod_{j \in \mathbb{Z}} \operatorname{Fit}_{R}\left(H^{j}(C)_{\mathrm{tor}}\right)^{(-1)^{j+1}}
$$

Here $\operatorname{det}(\lambda)$ is computed with respect to any choice of $R$-bases of $H^{a}(C) / H^{a}(C)_{\text {tor }}$ and $H^{a+1}(C) / H^{a+1}(C)_{\text {tor }}$ and $\operatorname{Fit}_{R}(M)$ is the Fitting ideal of an $R$-module $M$.

A.3. We require a variant of the (well-known) construction recalled in Section A.2 above. To describe this we fix a field $F$ of characteristic 0 , let $\Lambda$ denote either $F[G]$ or $F$ and suppose given a finite index set $\mathfrak{M}$ and for each $\mathfrak{m}$ in $\mathfrak{M}$ an exact sequence $\mathcal{E}_{\mathfrak{m}}$ of finitely generated (projective) $\Lambda$-modules $\cdots \rightarrow \mathcal{E}_{\mathfrak{m}}^{a-1} \rightarrow \mathcal{E}_{\mathfrak{m}}^{a} \rightarrow \mathcal{E}_{\mathfrak{m}}^{a+1} \rightarrow \cdots$ in which only finitely many modules $\mathcal{E}_{\mathfrak{m}}^{a}$ are non-zero. We regard each sequence $\mathcal{E}_{\mathfrak{m}}$ as an acyclic complex, with the module $\mathcal{E}_{\mathfrak{m}}^{a}$ placed in degree $a$, and write $\iota_{\mathcal{E}_{\mathfrak{m}}}$ for the natural morphism $\prod_{a \in \mathbb{Z}}\left[\mathcal{E}_{\mathfrak{m}}^{a}\right]_{\Lambda}^{(-1)^{a}} \rightarrow\left[\mathcal{E}_{\mathfrak{m}}\right]_{\Lambda} \rightarrow \mathbf{1}_{\Lambda}$ in $V(\Lambda)$.

Given a finite set of integers $\mathfrak{N}$ and for each $n$ in $\mathfrak{N}$ a finitely generated $\Lambda$-module $M_{n}$ we say that a morphism $t: \prod_{n \in \mathfrak{N}}\left[M_{n}\right]_{\Lambda}^{(-1)^{n}} \rightarrow \mathbf{1}_{\Lambda}$ in $V(\Lambda)$ is 'induced by $\left\{\mathcal{E}_{\mathfrak{m}}\right\}_{\mathfrak{m} \in \mathfrak{M}}$ ' if for each $n \in \mathfrak{N}$ one has $M_{n}=\mathcal{E}_{\mathfrak{m}_{n}}^{a_{n}}$ with $\mathfrak{m}_{n} \in \mathfrak{M}$ and $a_{n} \in \mathbb{Z}$ and if $n \neq n^{\prime}$ then either $\mathfrak{m}_{n} \neq \mathfrak{m}_{n^{\prime}}$ or $a_{n} \neq a_{n^{\prime}}$, and the morphism $t$ is equal to a composite of morphisms of the form $\iota_{\mathcal{E}_{\mathrm{m}}}$ together with standard commutativity $(X \cdot Y \rightarrow Y \cdot X)$, associativity $(X \cdot(Y \cdot Z) \rightarrow(X \cdot Y) \cdot Z)$ and contraction $\left(\operatorname{ev}_{W}\right)$ morphisms for suitable $\Lambda$-modules $X, Y, Z$ and $W$.

In the following result, we also use, for each finitely generated right $F[G]$-module $W$, the functor $X \mapsto X_{W}$ from $V(F[G])$ to $V(F)$ that is induced by the assignment $P \mapsto P_{W}:=W \otimes_{F[G]} P$ for each finitely generated (left) $F[G]$-module $P$.

Lemma A.1. Let $\mathfrak{N}$ be a finite set of integers, $\left\{M_{n}\right\}_{n \in \mathfrak{N}}$ a set of finitely generated $F[G]$-modules and $t: \prod_{n \in \mathfrak{N}}\left[M_{n}\right]_{F[G]}^{(-1)^{n}} \rightarrow \mathbf{1}_{F[G]}$ a morphism in $V(F[G])$ that is induced by a set of exact sequences of $F[G]$-modules. If $\phi: W \rightarrow W^{\prime}$ is an isomorphism of finitely generated right $F[G]$-modules, then the following diagram commutes

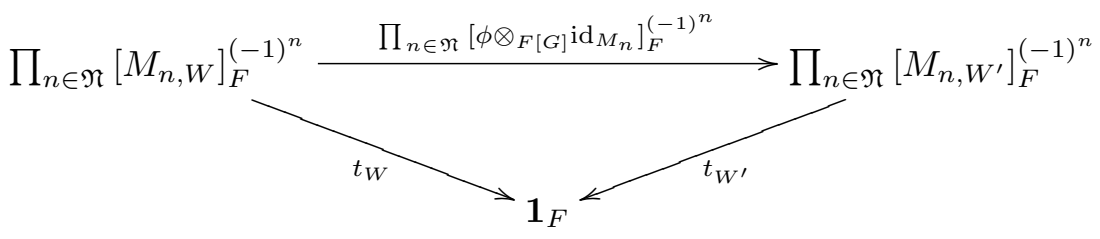

Proof. This is a straightforward consequence of the following fact: for any finitely generated $F[G]$-module $P$ there is a commutative diagram in $V(F)$ of the form

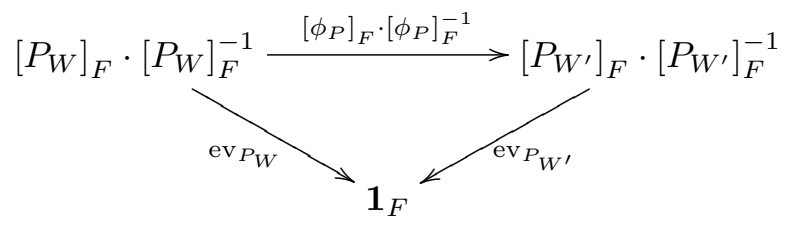


where $\phi_{P}:=\phi \otimes_{F[G]} \mathrm{id}_{P}$. Indeed, if we identify $V(F)$ with the category of graded line bundles on $F$ (as in Section A.1), then this diagram is equivalent to the obvious commutative diagram

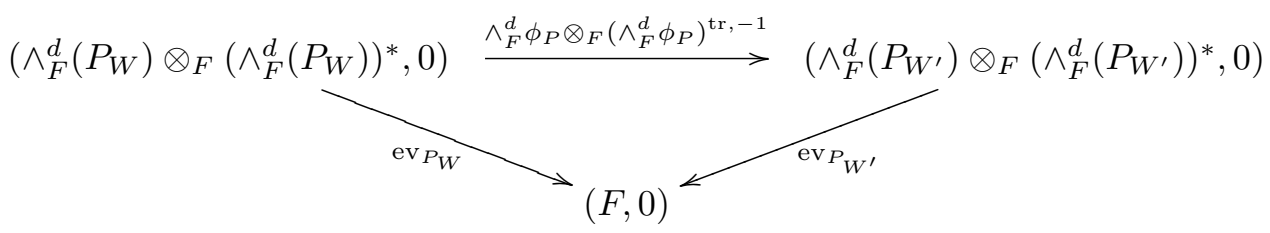

where we set $d:=\operatorname{dim}_{F}\left(P_{W}\right)=\operatorname{dim}_{F}\left(P_{W^{\prime}}\right)$ and write $\left(\wedge_{F}^{d} \phi_{P}\right)^{\text {tr }}$ for the transpose of the $F$-linear map $\wedge_{F}^{d} \phi_{P}$.

\section{Acknowledgment}

It is a pleasure to thank Alex Bartel for some stimulating discussions and Jan Nekovár for several very helpful comments.

\section{References}

[1] A. Bartel, On Brauer-Kuroda type relations of $S$-class numbers in dihedral extensions, J. Reine Angew. Math. 668 (2012), 211-244.

[2] A. Bartel and B. de Smit, Index formulae for integral Galois modules, submitted for publication.

[3] H. Bass, Algebraic K-theory, Benjamin, 1968.

[4] S. Bloch and K. Kato, L-functions and Tamagawa numbers of motives, in 'The Grothendieck Festschrift' vol. 1, Progress in Mathematics 86 (Birkhäuser, Boston, 1990), 333-400.

[5] D. Burns, Leading terms and values of equivariant motivic L-functions, Pure App. Math. Q. 6 (2010) (Special issue in honor of John Tate, Part II) 83-172.

[6] D. Burns and M. Flach, Tamagawa numbers for motives with (non-commutative) coefficients, Doc. Math. 6 (2001), 501-570.

[7] B. de Smit, Brauer-Kuroda relations for S-class numbers, Acta Arith. 98 (2001), $133-146$.

[8] P. Deligne, Le déterminant de la cohomologie, in Current Trends in Arithmetical Algebraic Geometry, Contemp. Math. 67, Amer. Math. Soc., 313-346, 1987.

[9] T. Dokchitser and V. Dokchitser, Regulator constants and the parity conjecture, Invent. Math. 178 (2009), 23-71.

[10] T. Dokchitser and V. Dokchitser, On the Birch-Swinnerton-Dyer Quotients modulo squares, Ann. Math. 172 (2010), 567-596.

[11] W.G. Dwyer and E.M. Friedlander, Algebraic and étale K-theory, Trans. Amer. Math. Soc. 292 (1985), 247-280.

[12] M. Flach, Euler characteristics in relative K-groups, Bull. London Math. Soc. 32 (2000), $272-284$.

[13] J.-M. Fontaine and B. Perrin-Riou, Autour des conjectures de Bloch et Kato: cohomologie galoisienne et valeurs de fonctions L, in: Motives (Seattle, 1991), Proc. Sympos. Pure Math. 55, Part 1 (Amer. Math. Soc., Providence, 1994), 599-706.

[14] M. Levine, The indecomposable $K_{3}$ of a field, Ann Sci. École Norm. Sup. 22 (1989), $255-344$.

[15] A.S. Merkurjev and A.A. Suslin, The $K_{3}$-group of a field, Izv. Nauk. SSSR 54 (1990), 339-356.

[16] C. Soulé, K-théorie des anneaux d'entiers de corps de nombres et cohomologie étale, Invent. Math. 55 (1979), 251-295. 
[17] J. Tate, Relations between $K_{2}$ and Galois Cohomology, Invent. Math. 36 (1976), 257-274.

[18] O. Venjakob, From the Birch and Swinnerton-Dyer Conjecture to non-commutative Iwasawa theory via the Equivariant Tamagawa Number Conjecture - a survey, in ' $L$-functions and Galois Representations', Proceedings of the 2004 Durham Symposium, 333-380, Cambridge University Press, 2007.

Department of Mathematics, King's College London, Strand Campus, London WC2R 2LS, UK

E-mail address: david.burns@kcl.ac.uk 
